\title{
Asymptotic normalization coefficients, spectroscopic factors, and direct radiative capture rates
}

\author{
A. M. Mukhamedzhanov, C. A. Gagliardi, and R. E. Tribble \\ Cyclotron Institute, Texas A\&M University, College Station, Texas 77843
}

(Received 14 August 2000; published 23 January 2001)

\begin{abstract}
We compare the use of asymptotic normalization coefficients (ANC's) and spectroscopic factors determined from peripheral transfer reactions for determining the overall normalization of peripheral direct radiative capture reaction processes. We demonstrate that ANC's provide a natural way to parametrize the rates of both peripheral transfer and direct capture reactions. Furthermore, ANC's inferred from one reaction may be used in the analysis of a second reaction without further knowledge regarding their origin, and independent measurements of a given ANC may be combined to give an overall "best value" in a straightforward manner. In contrast, a spectroscopic factor derived from analysis of a peripheral transfer reaction can only be used in subsequent calculations if one has detailed knowledge of the single-particle bound state orbital that was assumed when the spectroscopic factor was obtained.
\end{abstract}

DOI: 10.1103/PhysRevC.63.024612

PACS number(s): 25.40.Lw, 25.70.Hi, 25.55.Hp, 24.10.Eq

\section{INTRODUCTION}

In previous papers we suggested [1] and then demonstrated $[2,3]$ an indirect technique to determine direct capture rates at stellar energies from measurements of the corresponding asymptotic normalization coeffcients (ANC's) in peripheral transfer reactions. Direct capture reactions of astrophysical interest often involve systems where the binding energy of the captured particles is low, so the capture occurs through the tail of the nuclear overlap function in the corresponding two-body channel. The shape of this tail is dictated by the Coulomb interaction. Hence, the capture rate may be calculated accurately if one knows the amplitude of the tail, which is given by the ANC. ANC's may be measured in traditional nuclear reactions, such as peripheral nucleon transfer at energies above the Coulomb barrier, which have cross sections that are orders of magnitude larger than the direct capture reaction cross sections at astrophysical energies. ANC's have been studied in few-nucleon systems for many years, for example, the asymptotic $S$-wave and $D$-wave amplitudes in the deuteron, as well as the ANC's for $t,{ }^{3} \mathrm{He}$, and ${ }^{6} \mathrm{Li}$. See [4-6] for reviews. But the relation between peripheral transfer reaction cross sections and ANC's and the importance of this relation as an indirect technique to measure astrophysical radiative capture rates have only recently been stressed. Usually the distorted-wave Born approximation (DWBA) is used to analyze peripheral transfer reactions. However, in conventional DWBA, the transfer reaction amplitude is parametrized in terms of spectroscopic factors rather than ANC's. We articulate here the difference between and the relationship that connects ANC's and spectroscopic factors. We also address an important consistency issue: the parametrization of a DWBA cross section in terms of spectroscopic factors is most appropriate for nonperipheral transfer reactions, but in such cases, several of the assumptions underlying traditional DWBA treatments are questionable.

\section{ANC's AND SPECTROSCOPIC FACTORS}

The overlap function of the bound state wave functions for particles $A, p$, and $B$, where $B=(A p)$ is the bound state of nucleus $A$ and proton $p$, is given by

$$
\begin{aligned}
I_{A p}^{B}(\mathbf{r})= & (A+1)^{1 / 2}\left\langle\varphi_{A}\left(\zeta_{A}\right) \varphi_{p}\left(\zeta_{p}\right) \mid \varphi_{B}\left(\zeta_{A}, \zeta_{p} ; \mathbf{r}\right)\right\rangle \\
= & \sum_{l_{B} m_{l_{B}} j_{B} m_{j_{B}}}\left\langle J_{A} M_{A} j_{B} m_{j_{B}} \mid J_{B} M_{B}\right\rangle \\
& \times\left\langle J_{p} M_{p} l_{B} m_{l_{B}} \mid j_{B} m_{j_{B}}\right\rangle i^{l_{B}} Y_{l_{B} m_{l_{B}}}(\hat{\mathbf{r}}) I_{A p l_{B} j_{B}}^{B}(r) .
\end{aligned}
$$

For each nucleus $\varphi$ represents the bound state wave function with $\zeta$ being a set of internal coordinates including spinisospin variables, and $J$ and $M$ are the spin and spin projection. Also $\mathbf{r}$ is the radius vector connecting the center of mass of nucleus $A$ with $p, \hat{\mathbf{r}}=\mathbf{r} / r, j_{B}, m_{j_{B}}$ are the total angular momentum of the proton in the nucleus $B=(A p)$ and its projection, $l_{B}, m_{l_{B}}$ are the orbital angular momentum of the relative motion of particles $A$ and $p$ in the bound state $B=(A p)$ and its projection, and $I_{A p l_{B} j_{B}}^{B}(r)$ is the radial overlap function. The antisymmetrization factor, $(A+1)^{1 / 2}$, due to identical nucleons has been absorbed in the radial overlap function. The summation over $l_{B}$ and $j_{B}$ is carried out over the values allowed by angular momentum and parity conservation in the virtual process $B \rightarrow A+p$.

The overlap function is the projection of the state $\varphi_{B}$ onto the two-body channel $\varphi_{A} \varphi_{p}$. This projection is not an eigenfunction of any Hermitian Hamiltonian and is not directly associated with a probability. Hence, the overlap function is not normalized to unity. The square of the norm of the overlap function

$$
S_{A p}=\int d \mathbf{r}\left[I_{A p}^{B}(\mathbf{r})\right]^{2}
$$

is, by definition, the spectroscopic factor. The asymptotic behavior of the radial overlap function is given by

$$
I_{A p l_{B} j_{B}}^{B}(r) \stackrel{r>R_{N}}{\rightarrow} C_{A p l_{B} j_{B}}^{B} \frac{W_{-\eta_{B}, l_{B}+1 / 2}\left(2 \kappa_{B} r\right)}{r} .
$$


Here $C_{A p l_{B} j_{B}}^{B}$ is the asymptotic normalization coefficient defining the amplitude of the tail of the radial overlap function $I_{A p l_{B} j_{B}}^{B}(r)[4,1], W_{-\eta_{B}, l_{B}+1 / 2}\left(2 \kappa_{B} r\right)$ is the Whittaker function describing the asymptotic behavior of the Schrödinger equation solution for two charged particles at negative energy, $\kappa_{B}=\sqrt{2 \mu_{A p} \varepsilon_{B}}$ is the wave number of the bound state $B=(A p), \mu_{A p}$ is the reduced mass of particles $A$ and $p$, and $\eta_{B}=Z_{A} Z_{p} \mu_{A p} / \kappa_{B}$ is the Coulomb parameter of the bound state $(A p)$. With this definition, the ANC includes the antisymmetrization factor. We do this because our goal is to relate experimental cross sections for peripheral transfer and radiative capture reactions, and the experimental measurements are incapable of separating the effects of antisymmetrization from those due the underlying potential. Note that this differs from the typical convention in few-nucleon studies, where the goal often is to investigate the sensitivity of calculated quantities to assumptions regarding the underlying $N N$ and few-nucleon forces $[4,7,8]$.

There is a principal difference between the ANC and the spectroscopic factor. The radial overlap function dies off exponentially as $r \rightarrow \infty$, so the main contribution to the radial integral (2) comes from the nuclear interior, $r<R_{N}$. Thus, the spectroscopic factor is defined predominantly by the behavior of the radial overlap function within the nucleus. In contrast, the ANC describes the peripheral properties of the overlap function since it defines the amplitude of the tail. The ANC is, of course, coupled to the internal behavior of the nuclear wave function, and Green's function techniques permit it to be expressed as an integral over the nuclear core alone. However, calculations have demonstrated that, for cases of astrophysical interest, the resultant integral is extremely sensitive to the form of the $N N$ interaction that is assumed, severely limiting the predictive power of this approach $[8-10]$.

The ANC can also be defined through the residue at the pole of the elastic scattering amplitude corresponding to the bound state $B=(A p)_{l_{B} j_{B}}[11,12]$

$$
F_{l_{B} j_{B}}(k) \stackrel{k \rightarrow i \kappa_{B}}{\rightarrow}(-1)^{l_{B}} i e^{i \pi \eta_{B}} \frac{\left|C_{A p l_{B} j_{B}}^{B}\right|^{2}}{k-i \kappa_{B}} .
$$

Here $F_{l_{B} j_{B}}(k)$ is the partial scattering amplitude in the channel $\left(l_{B}, j_{B}\right)$, and $k$ is the relative momentum of particles $A$ and $p$. Equation (4) is a model-independent definition of the ANC because it does not depend on any specific assumed interaction. Furthermore, Eq. (4) even remains true for the pole corresponding to a Breit-Wigner resonance $[11,12]$.

To find the overlap function one has to solve an infinite number of coupled integro-differential equations. Because of technical difficulties, different approximations are used, including microscopic ones, such as the shell-model or resonating group method (RGM) and simpler ones, such as the single-particle approach. In the shell model and RGM, the spectroscopic factor is a model-dependent quantity that is sensitive to the adopted potential and to the truncations inherent in the calculations. In general the spectroscopic factor can differ from unity since it depends on the contribution of an infinite number of channels coupled to the two-body channel $(A p)_{l_{B} j_{B}}$ plus antisymmetrization effects, but in the microscopic approach, the deviation of the spectroscopic factor from 1 is caused only by antisymmetrization effects that engage different nonorthogonal channels. Due to these antisymmetrization effects, the spectroscopic factor calculated in microscopic approaches can be larger than 1 .

In the single-particle approach, the radial overlap function is approximated by a single-particle overlap function

$$
I_{A p l_{B} j_{B}}^{B}(r) \approx I_{A p l_{B} j_{B}}^{B(s p)}(r)=\left[S_{l_{B} j_{B}}^{(s p)}\right]^{1 / 2} \varphi_{n_{B} l_{B} j_{B}}(r),
$$

where $\varphi_{n_{B} l_{B} j_{B}}(r)$ is the normalized single-particle radial wave function of the bound state $(A p)$ calculated in an adopted $A-p$ interaction potential (often Woods-Saxon) and $n_{B}$ is the principal quantum number. This approximation is usually used in DWBA analysis of experimental data. Since the squares of the norms of the overlap function and the radial bound-state wave function are, correspondingly, the spectroscopic factor $S_{l_{B} j_{B}}$ and unity, the single-particle spectroscopic factor $S_{l_{B} j_{B}}^{(s p)}$ in Eq. (5) will equal the spectroscopic factor $S_{l_{B} j_{B}}$ if the bound-state wave function and the overlap function have very similar radial behavior both in the nuclear interior and exterior. However, for $r<R_{N}$ where both $I$ and $\varphi$ have most of their probability, the radial dependence of the overlap function and single-particle wave function, a priori, are different because the overlap function is a many-particle object, whereas the single-particle wave function is a solution of the single-particle Schrödinger equation. Thus, in general $S_{l_{B} j_{B}}^{(s p)}$ does not coincide with the microscopically calculated spectroscopic factor $S_{l_{B} j_{B}}$. Nonetheless, for $r>R_{N}$, the radial dependencies of $I_{A p l_{B} j_{B}}^{B}(r)$ and $\varphi_{n_{B} l_{B} j_{B}}(r)$ are the same, and they differ only by their overall normalizations. The asymptotic behavior of the radial overlap function is given by Eq. (3), and the asymptotic normalization of the radial bound-state wave function is defined as

$$
\varphi_{n_{B} l_{B} j_{B}}(r) \stackrel{r>R_{N}}{\rightarrow} b_{l_{B} j_{B}} \frac{W_{-\eta_{B}, l_{B}+1 / 2}\left(2 \kappa_{B} r\right)}{r} .
$$

By the proper choice of $S_{l_{B} j_{B}}^{(s p)}$, one can make Eq. (5) exact for $r>R_{N}$. Then, comparing Eqs. (3) and (6) gives the relationship connecting the single-particle spectroscopic factor, the nuclear ANC, and the single-particle ANC $b_{l_{B} j_{B}}$

$$
S_{l_{B} j_{B}}^{(s p)}=\frac{\left(C_{A p l_{B} j_{B}}^{B}\right)^{2}}{\left(b_{l_{B} j_{B}}\right)^{2}} \text {. }
$$

While the ANC is an experimentally measurable quantity, the single-particle ANC $b_{l_{B} j_{B}}$ is not. Hence, the singleparticle spectroscopic factor, when defined by Eq. (7), is model dependent. Its model dependence comes through the single-particle ANC $b_{l_{B} j_{B}}$, which is a function of the geometric parameters, radius $r_{0}$ and diffuseness $a$, of the WoodsSaxon potential conventionally used as a single-particle potential. Furthermore, note that, unlike $S_{l_{B} j_{B}}$ as defined in Eq. 
(2), $S_{l_{B} j_{B}}^{(s p)}$ in Eq. (7) is actually a property of the peripheral part of the nucleon overlap function.

To illuminate the differences pointed out above, compare the single-particle potential approach and the microscopic RGM. In the simple one-channel RGM, the overlap function is given by

$$
\begin{aligned}
I_{A p}^{B(R G M)}(\mathbf{r})= & \left\langle\mathcal{A}\left[\varphi_{A}\left(\zeta_{A}\right) \varphi_{p}\left(\zeta_{p}\right) \delta\left(\boldsymbol{\rho}^{\prime}-\mathbf{r}\right)\right]\right| \mathcal{A} \\
& \left.\times\left[\varphi_{A}\left(\zeta_{A}\right) \varphi_{p}\left(\zeta_{p}\right) \delta\left(\boldsymbol{\rho}-\boldsymbol{\rho}^{\prime}\right) \chi_{A p}(\boldsymbol{\rho})\right]\right\rangle \\
= & \int d \boldsymbol{\rho} \mathcal{K}(\boldsymbol{\rho}, \mathbf{r}) \chi_{A p}(\boldsymbol{\rho}),
\end{aligned}
$$

where $\mathcal{A}$ denotes antisymmetrization between nucleons of nuclei $A$ and $p, \chi_{A p}(\mathbf{r})$ is the wave function of the relative motion of $A$ and $p$ in the RGM, and $\mathcal{K}$ is the integral operator whose kernel is given by

$$
\mathcal{K}(\boldsymbol{\rho}, \mathbf{r})=\left\langle\mathcal{A}\left[\varphi_{A} \varphi_{p} \delta\left(\boldsymbol{\rho}^{\prime}-\mathbf{r}\right)\right] \mid \mathcal{A}\left[\varphi_{A} \varphi_{p} \delta\left(\boldsymbol{\rho}-\boldsymbol{\rho}^{\prime}\right)\right]\right\rangle .
$$

The operator $\mathcal{K}$ is different from the unit operator due to the presence of $\mathcal{A}$. At large distances the antisymmetrization effects become negligible, so

$$
\mathcal{K}(\boldsymbol{\rho}, \mathbf{r}) \stackrel{r \rightarrow \infty}{\rightarrow} \delta(\boldsymbol{\rho}-\mathbf{r}),
$$

and

$$
I_{A p l_{B} j_{B}}^{B(R G M)}(r) \stackrel{r \rightarrow \infty}{\rightarrow} \chi_{A p l_{B} j_{B}}(r) \stackrel{r \rightarrow \infty}{\rightarrow} C_{A p l_{B} j_{B}}^{B(R G)} \frac{W_{-\eta_{B}, l_{B}+1 / 2}\left(2 \kappa_{B} r\right)}{r} .
$$

Here $\chi_{A p l_{B} j_{B}}(r)$ is the radial part of $\chi_{A p}(\mathbf{r})$. Thus the tails of the radial overlap function and the RGM wave function of the relative motion of $A$ and $p$ coincide. Note that, while each bound-state wave function $\varphi_{\nu}$ is normalized to unity, the RGM wave function of the relative $A-p$ motion $\chi_{A p}(\mathbf{r})$ is not normalized to unity. Its norm does not possess any physical meaning, but the amplitude of the tail is given by the ANC. Thus, we have three different radial functions-the RGM overlap function $I_{A p l_{B} j_{B}}^{B(R G M)}(r)$, the RGM wave function of the relative motion $\chi_{A p l_{B} j_{B}}(r)$, and the single-particle overlap function $\left[S_{l_{B} j_{B}}^{(s p)}\right]^{1 / 2} \varphi_{n_{B} l_{B} j_{B}}(r)$-which have the same asymptotic behavior. However, their radial shapes may be quite different inside nucleus $B$. Antisymmetrization effects become important, so operator $\mathcal{K}$ is not unity and the shapes of $I_{A p l_{B} j_{B}}^{B(R G M)}(r)$ and $\chi_{A p l_{B} j_{B}}(r)$ are different [13]. Furthermore, being many-particle objects, both $I$ and $\chi$ may have different behavior in the internal region from the single-particle wave function $\varphi_{n_{B} l_{B} j_{B}}(r)$. In Fig. 1 we show the radial RGM [13] and single-particle overlap functions for ${ }^{8} \mathrm{~B} \rightarrow{ }^{7} \mathrm{Be}+p$ as functions of $r$. All functions were calculated for the channel spin $I=2$. The single-particle bound-state wave functions were calculated for different sets of geometric parameters $\left(r_{0}, a\right)$ of the Woods-Saxon potential. The single-particle spectroscopic factors are chosen to satisfy the asymptotic condition (7) that provides the same asymptotic behavior for

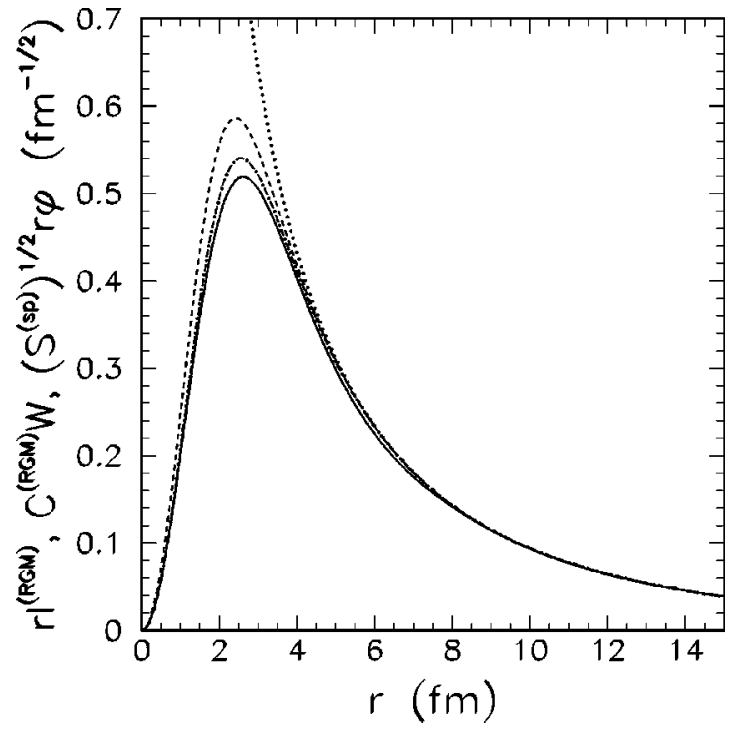

FIG. 1. The calculated radial RGM overlap function $r I^{(R G M)}(r)$ for ${ }^{8} \mathrm{~B} \rightarrow{ }^{7} \mathrm{Be}+p$ [13] (solid line), the asymptotics of the overlap function $C^{(R G M)} W(r)$ [13] (dotted line), and the single-particle overlap functions $\left[S^{(s p)}\right]^{1 / 2} r \varphi(r)$ : dot-dashed line for $r_{0}$ $=1.25 \mathrm{fm}$ and $a=0.65 \mathrm{fm}$ and dashed line for $r_{0}=1.20 \mathrm{fm}$ and $a=0.60 \mathrm{fm}$. $\left[S^{(s p)}\right]^{1 / 2} r \varphi(r)$ for $r_{0}=1.3 \mathrm{fm}$ and $a=0.675 \mathrm{fm}$ (not shown) agrees with $r I^{(R G M)}(r)$ to within $1 \%$ at all radii. All functions were calculated for the channel spin $I=2$.

the microscopic and single-particle overlap functions. As one can see, the microscopic and single-particle overlap functions have different behavior in the nuclear interior. We were able to find geometric parameters $\left(r_{0}=1.3 \mathrm{fm}\right.$ and $a$ $=0.675 \mathrm{fm}$ ) for which the RGM and single-particle overlap functions coincide to within $1 \%$ at all radii, but it is impossible to find such a bound-state Woods-Saxon potential without prior knowledge of the microscopic overlap function.

Thus any spectroscopic factor introduced by Eq. (5) must be utilized in any further analysis together with its corresponding single-particle wave function $\varphi_{n_{\alpha^{\prime}} l_{\alpha} j_{\alpha}}\left(r_{\alpha}\right)$.

\section{TRANSFER REACTION MEASUREMENTS}

Previously [14] we have described the technique of determining ANC's from peripheral transfer reactions. The ANC's determined from transfer reactions can then be used to calculate the astrophysical factors for radiative capture processes. Here we elucidate the difference between the extraction of ANC's and spectroscopic factors determined from the transfer reactions, and in the following section we demonstrate that the ANC is the natural quantity to use to calculate astrophysical factors.

Consider the proton transfer reaction

$$
a+A \rightarrow b+B,
$$

where $a=b+p, B=A+p$. Traditionally, the DWBA cross section used to analyze the experimental angular distribution is given by 


$$
\frac{d \sigma}{d \Omega}=\sum_{j_{B} j_{a}} S_{A p l_{B} j_{B}} S_{b p l_{a} j_{a}} \sigma_{l_{B} j_{B} l_{a} j_{a}}^{D W} .
$$

Originally this parametrization of the DWBA cross section in terms of spectroscopic factors was designed for the determination of spectroscopic factors by normalization of the calculated DWBA reduced cross section $\sigma^{D W}$ to the experimental differential cross section in the main peak of the angular distribution generated by the proton transfer mechanism. However, Eq. (13) is derived by using approximation (5) for the overlap integrals corresponding to the two vertices describing the virtual decay $a \rightarrow b+p$ and synthesis $A+p$ $\rightarrow B$ in the reaction (12). Thus, the spectroscopic factors in Eq. (13) are actually single-particle spectroscopic factors. This distinction has often been neglected in the literature, and many authors have equated experimental spectroscopic factors extracted from fits using Eq. (13) to theoretical spectroscopic factors defined according to Eq. (2), such as those in Ref. [15].

The spectroscopic factor in Eq. (2), being a physical characteristic of the behavior of the overlap function in the nuclear interior, can be extracted unambiguously only if the reaction is not peripheral. But for reactions dominated by the nuclear interior, the simple approximation made in Eq. (5) and assumed in Eq. (13) is not valid since antisymmetrization and many-body effects are important. For example, when using the microscopic RGM approach the overlap function has to be replaced by $\mathcal{K}_{\chi_{A p}}(\mathbf{r})$, which in the nuclear interior may differ significantly from $\left[S_{l_{B} j_{B}}^{(s p)}\right]^{1 / 2} \varphi_{n_{B} l_{B} j_{B}}(r)$. Furthermore, in the nuclear interior the initial-and final-state wave functions cannot be written in factorized form as overlap functions times distorted waves. An additional complication of traditional DWBA is the first-order perturbation approach in which the transition operator $\Delta V$ sandwiched by the initial- and final-state wave functions is assumed to be small. This is certainly not guaranteed for reactions that are not peripheral [16].

For peripheral reactions, Eq. (7) makes Eq. (5) exact in the region of interest. Substituting it in Eq. (13), we find

$$
\frac{d \sigma}{d \Omega}=\sum_{j_{B} j_{a}}\left(C_{A p l_{B} j_{B}}^{B}\right)^{2}\left(C_{b p l_{a} j_{a}}^{a}\right)^{2} R_{l_{B} j_{B} l_{a} j_{a}}
$$

where

$$
R_{l_{B} j_{B} l_{a} j_{a}}=\frac{\sigma_{l_{B} j_{B} l_{a} j_{a}}^{D W}}{b_{A p l_{B} j_{B}}^{2} b_{b p l_{a} j_{a}}^{2}} .
$$

Equation (6) implies $\sigma_{l_{B} j_{B} l_{a} j_{a}}^{D W} \propto b_{A p l_{B} j_{B}}^{2} b_{b p l_{a} j_{a}}^{2}$ for peripheral reactions [14], so $R$ is nearly independent of $b_{A p l_{B} j_{B}}$ and $b_{b p l_{a} j_{a}}$. Equation (14) represents the basis for the determination of the product of the ANC's $\left(C_{A p l_{B} j_{B}}^{B}\right)^{2}\left(C_{b p l_{a} j_{a}}^{a}\right)^{2}$ involved in a reaction. In transfer reactions, two possibilities can occur. If $a$ and $B$ are the same nuclei, i.e., we have an elastic exchange reaction, the DWBA cross section is expressed in terms of $\left(C_{b p l_{a} j_{a}}^{a}\right)^{4}$ and this ANC can be deter- mined by normalizing the DWBA cross section to the experimental one [14]. If $a$ and $B$ are different nuclei, then to determine $C_{A p l_{B} j_{B}}^{B}$, one has to know $C_{b p l_{a} j_{a}}^{a}$ from an independent measurement. Since the ANC is a modelindependent quantity, the ANC $C_{b p l_{a} j_{a}}^{a}$ found from any other reliable experimental source-including those found from transfer reactions involving light or heavy ions or from analysis of elastic scattering — can be used in the subsequent DWBA analysis.

As a specific example, consider use of the ${ }^{14} \mathrm{~N}\left({ }^{7} \mathrm{Be},{ }^{8} \mathrm{~B}\right){ }^{13} \mathrm{C}$ reaction to determine the ANC for ${ }^{8} \mathrm{~B}$ $\rightarrow{ }^{7} \mathrm{Be}+p$ [17]. Since the DWBA cross section is parametrized in terms of the product of the ANC's for proton removal from ${ }^{14} \mathrm{~N}$ and ${ }^{8} \mathrm{~B}$, to determine the ANC for ${ }^{8} \mathrm{~B}$ $\rightarrow{ }^{7} \mathrm{Be}+p$, one has to know the ANC for ${ }^{14} \mathrm{~N} \rightarrow{ }^{13} \mathrm{C}+p$. Two different reactions, ${ }^{13} \mathrm{C}\left({ }^{14} \mathrm{~N},{ }^{13} \mathrm{C}\right){ }^{14} \mathrm{~N} \quad[18]$ and ${ }^{13} \mathrm{C}\left({ }^{3} \mathrm{He}, d\right){ }^{14} \mathrm{~N}$ [19], have been used to determine this. Since ${ }^{13} \mathrm{C}\left({ }^{14} \mathrm{~N},{ }^{13} \mathrm{C}\right){ }^{14} \mathrm{~N}$ is an elastic exchange, the DWBA amplitude contains identical initial and final bound-state wave functions and, hence, the DWBA cross section is normalized in terms of $\left[C_{13}^{14} \mathrm{~N} p\right]^{4}$. (For simplicity the quantum numbers specifying the virtual decay have been dropped.) No other ANC's are required. An additional advantage in this case is that the optical potentials in the entrance and exit channels are identical. The DWBA cross section for ${ }^{13} \mathrm{C}\left({ }^{3} \mathrm{He}, d\right){ }^{14} \mathrm{~N}$ is proportional to $\left[C_{13}^{14} \mathrm{~N} p\right]^{2}\left[C_{d p}^{3}\right]^{3}$. Hence, from normalization of the DWBA reduced cross section to the experimental one, we can determine this product. However, the ANC for ${ }^{3} \mathrm{He}$ $\rightarrow d+p$ is well established, so we can obtain the ANC for ${ }^{14} \mathrm{~N} \rightarrow{ }^{13} \mathrm{C}+p$. Since the ANC is a model-independent nuclear characteristic, it is independent of the reaction used to extract it if the reaction is peripheral and optical potentials are properly chosen. In fact, the analysis of both reactions gave consistent results for the dominant $p_{1 / 2}$ component in ${ }^{14} \mathrm{~N}:\left[C_{13}^{14} \mathrm{~N} p\right]^{2}=18.6 \pm 1.2 \mathrm{fm}^{-1}$ from the elastic exchange reaction [18] and $17.8 \pm 1.3 \mathrm{fm}^{-1}$ from the $\left({ }^{3} \mathrm{He}, d\right)$ reaction [19]. In subsequent analysis we can adopt the weighted average of the two values in order to extract the ANC for the virtual decay ${ }^{8} \mathrm{~B} \rightarrow{ }^{7} \mathrm{Be}+p$ from the ${ }^{14} \mathrm{~N}\left({ }^{7} \mathrm{Be},{ }^{8} \mathrm{~B}\right){ }^{13} \mathrm{C}$ reaction.

In contrast, consider the use of the spectroscopic factors for ${ }^{14} \mathrm{~N} \rightarrow{ }^{13} \mathrm{C}+p$ in these reactions. Reference [18] demonstrates that the spectroscopic factor derived from the ${ }^{13} \mathrm{C}\left({ }^{14} \mathrm{~N},{ }^{13} \mathrm{C}\right){ }^{14} \mathrm{~N}$ reaction varies by more than a factor of 2 when the single-particle bound-state potential parameters $\left(r_{0}, a\right)$ used in the DWBA calculations are adjusted within normal ranges. Reference [19] finds an even broader variation of the spectroscopic factor in the ${ }^{13} \mathrm{C}\left({ }^{3} \mathrm{He}, d\right){ }^{14} \mathrm{~N}$ reaction study. Thus, use of different single-particle potentials for the two reactions would lead to different values of the spectroscopic factors for the configuration ${ }^{13} \mathrm{C}+p$ in ${ }^{14} \mathrm{~N}$. Each of these spectroscopic factors could only be used in the following analysis of the ${ }^{14} \mathrm{~N}\left({ }^{7} \mathrm{Be},{ }^{8} \mathrm{~B}\right){ }^{13} \mathrm{C}$ reaction if the proton bound-state wave function in ${ }^{14} \mathrm{~N}$ is calculated with exactly the same bound-state potential parameters used to derive the spectroscopic factor. Consequently, it would not be possible to combine the results of the two independent mea- 
surements of the ${ }^{14} \mathrm{~N} \rightarrow{ }^{13} \mathrm{C}+p$ system in a simple way, as can be done for the ANC analysis.

\section{DIRECT CAPTURE REACTIONS}

As proposed in [1], the ANC can be used to calculate direct radiative capture at low energies leading to a loosely bound final nucleus

$$
A+p \rightarrow B+\gamma
$$

The direct capture amplitude in this case is given by

$$
M^{D C}=\lambda\left\langle\varphi_{B}\left(\zeta_{A}, \zeta_{p} ; \mathbf{r}\right)|\hat{O}| \varphi_{A}\left(\zeta_{A}\right) \varphi_{p}\left(\zeta_{p}\right) \psi_{i}^{+}(\mathbf{r})\right\rangle \text {, }
$$

where $\lambda$ is a kinematic factor, $\hat{O}$ is the electromagnetic transition operator, and $\psi_{i}^{+}$is the scattering wave function in the initial state. Note that Eq. (17) holds only for peripheral capture reactions. For nonperipheral capture, the amplitude is more complicated because nucleon degrees of freedom should be treated explicitly when writing down the electromagnetic operator and antisymmetrization effects between the incident proton and the nucleons of target $A$ are important. Here we assume that the capture is peripheral so that the antisymmetrization operator has been dropped and the electromagnetic operator can be taken in the two-body approximation.

An additional simplification occurs in the limit $E \rightarrow 0$, often the energy region of greatest interest in nuclear astrophysics. In this case, the initial scattering wave $\psi_{i}^{+}$becomes a Coulomb wave. This eliminates ambiguities associated with the assumed form of the $p$-nucleus interaction. For example, when calculating the ${ }^{16} \mathrm{O}(p, \gamma){ }^{17} \mathrm{~F}$ direct capture rate, the result changed by less than $2 \%$ for $E_{c . m .}<1 \mathrm{MeV}$ when the exact initial scattering wave function was replaced by the corresponding Coulomb wave function [2]. Similar insensitivity to the $p$-nucleus interaction has been found for ${ }^{7} \mathrm{Be}(p, \gamma){ }^{8} \mathrm{~B}[1]$ and $\alpha(d, \gamma){ }^{6} \mathrm{Li}[20]$ at energies relevant to astrophysics.

After integration and summation over the internal degrees of freedom, we obtain

$$
\sigma^{D C}=\sum_{j_{B}}\left(C_{A p l_{B} j_{B}}^{B}\right)^{2} \frac{\widetilde{\sigma}_{l_{B} j_{B}}^{D C}}{b_{A p l_{B} j_{B}}^{2}}
$$

for the direct radiative capture cross section. We assume, for simplicity, that only one $l_{B}$ contributes to the matrix element. Equation (18) can be rewritten in a form similar to that of Eq. (14),

$$
\sigma^{D C}=\sum_{j_{B}}\left(C_{A p l_{B} j_{B}}^{B}\right)^{2} R_{l_{B} j_{B}}^{D C}
$$

where

$$
R_{l_{B} j_{B}}^{D C}=\frac{\widetilde{\sigma}^{D C}}{b_{A p l_{B} j_{B}}^{2}} .
$$

For peripheral direct capture, Eq. (6) ensures that $\widetilde{\sigma}_{l_{B} j_{B}}^{D C}$ $\propto b_{A p l_{B} j_{B}}^{2}$. Thus, $R_{l_{B} j_{B}}^{D C}$ is practically independent of $b_{A p l_{B} j_{B}}$ and hence, of the single-particle potential used to calculate the bound-state wave function $B=(A p)$. Moreover, for electric transitions $R_{l_{B} j_{B}}^{D C}$ does not depend on $j_{B}$, which allows us to rewrite Eq. (19) as

$$
\sigma^{D C}=\left[\sum_{j_{B}}\left(C_{A p l_{B} j_{B}}^{B}\right)^{2}\right] R_{l_{B}}^{D C} .
$$

Thus, the overall normalization of the cross section of the peripheral direct capture is expressed in terms of the ANC's, which are the only unknown quantities in Eq. (21). Once the ANC's have been determined, they can be used to calculate the direct capture amplitude. We can, of course, rewrite $\sigma^{D C}$ in the equivalent form

$$
\sigma^{D C}=\sum_{j_{B}} S_{A p l_{B} j_{B}}^{B} \widetilde{\sigma}_{l_{B} j_{B}}^{D C}
$$

expressing the direct capture cross section in terms of spectroscopic factors. Such a parametrization is the conventional one in the potential approach when the overlap function is approximated by Eq. (5). However, since $\widetilde{\sigma}_{l_{B} j_{B}}^{D C} \propto b_{A p l_{B} j_{B}}^{2}$, when calculating the direct capture cross section by Eq. (22), the spectroscopic factors must be utilized along with the parameters of the bound-state potential well used in the prior analysis of the transfer reaction.

This procedure may be applied to the specific example of calculating the astrophysical $S$ factor for the radiative capture reaction ${ }^{7} \mathrm{Be}(p, \gamma){ }^{8} \mathrm{~B}$ as $E \rightarrow 0$. Since this process is highly peripheral at low energies, the overall normalization of the dominant $E 1$ transition is solely defined by the two ANC's for ${ }^{8} \mathrm{~B} \rightarrow{ }^{7} \mathrm{Be}+p$ corresponding to $j_{B}=3 / 2$ and $1 / 2$. These ANC's have been measured in two different proton transfer reactions: ${ }^{10} \mathrm{~B}\left({ }^{7} \mathrm{Be},{ }^{8} \mathrm{~B}\right){ }^{9} \mathrm{Be}$ [3] and ${ }^{14} \mathrm{~N}\left({ }^{7} \mathrm{Be},{ }^{8} \mathrm{~B}\right){ }^{13} \mathrm{C}$ [17]. The results are in good agreement and either one, or the appropriate weighted average, can be used when calculating $S_{17}(E)$ at low $E$. However, if one tries to use spectroscopic factors rather than ANC's, the "preexisting history" becomes crucial. To get the correct answer requires using each spectroscopic factor together with the corresponding singleparticle bound state for ${ }^{8} \mathrm{~B}$ that was assumed when the spectroscopic factor was extracted from experimental data. This makes it extremely difficult to combine the results of the separate measurements to obtain a final "best value," especially when some uncertainties in the experiments are correlated and others are independent, as in Refs. $[3,17]$.

\section{CONCLUSION}

We have demonstrated that ANC's provide a natural way to parametrize the rates of both peripheral transfer reactions and direct radiative capture reactions. Furthermore, ANC's inferred from one reaction may be used in the analysis of a second reaction without further knowledge regarding their origin, and independent measurements of a given ANC may be combined to give an overall "best value" in a straight- 
forward manner. In contrast, a spectroscopic factor derived from analysis of a peripheral transfer reaction can only be used in subsequent calculations if one has detailed knowledge of the single-particle bound-state orbital that was assumed when the spectroscopic factor was obtained. This makes it difficult to combine together the results of independent measurements, especially when they include both correlated and uncorrelated uncertainties. In this paper, we have specialized to the case of proton transfer and capture reactions. However, the same formalism and conclusions apply for alpha [21] and neutron [22] transfer and capture reactions so long as they also obey similar constraints on peripherality.

\section{ACKNOWLEDGMENTS}

We thank A. Csoto for providing us the numerical results from his RGM calculations for ${ }^{8} \mathrm{~B}$ in Ref. [13]. This work was supported in part by the U.S. Department of Energy under Grant No. DE-FG03-93ER40773 and by the Robert A. Welch Foundation.
[1] H.M. Xu, C.A. Gagliardi, R.E. Tribble, A.M. Mukhamedzhanov, and N.K. Timofeyuk, Phys. Rev. Lett. 73, 2027 (1994).

[2] C.A. Gagliardi et al., Phys. Rev. C 59, 1149 (1999).

[3] A. Azhari, V. Burjan, F. Carstoiu, H. Dejbakhsh, C.A. Gagliardi, V. Kroha, A.M. Mukhamedzhanov, L. Trache, and R.E. Tribble, Phys. Rev. Lett. 82, 3960 (1999).

[4] L.D. Blokhintsev, I. Borbely, and E.I. Dolinskii, Fiz. Elem. Chastits At. Yadra 8, 1189 (1977) [Sov. J. Part. Nucl. 8, 485 (1977)].

[5] M.P. Locher and T. Mizutani, Phys. Rep. 46, 43 (1978).

[6] D.R. Lehman, Colloq. Phys. 51, 15 (1990).

[7] B.F. Gibson and D.R. Lehman, Phys. Rev. C 29, 1017 (1984).

[8] A.M. Mukhamedzhanov and N.K. Timofeyuk, Yad. Fiz. 51, 679 (1990) [Sov. J. Nucl. Phys. 51, 431 (1990)].

[9] T. Kajino, Nucl. Phys. A460, 559 (1986).

[10] B.K. Jennings, Phys. Rev. C 62, 027602 (2000).

[11] A.I. Baz', Ya.B. Zel'dovich, and A.M. Perelomov, Nonrelativistic Quantum Theory of Scattering, Reactions and Decays (Nauka, Moscow, 1975).

[12] A.M. Mukhamedzhanov and R.E. Tribble, Phys. Rev. C 59, 3418 (1999).
[13] A. Csoto, Phys. Rev. C 61, 037601 (2000).

[14] A.M. Mukhamedzhanov et al., Phys. Rev. C 56, 1302 (1997).

[15] S. Cohen and D. Kurath, Nucl. Phys. A101, 1 (1967).

[16] I.J. Thompson, Comput. Phys. Rep. 7, 167 (1988).

[17] A. Azhari, V. Burjan, F. Carstoiu, C.A. Gagliardi, V. Kroha, A.M. Mukhamedzhanov, X. Tang, L. Trache, and R.E. Tribble, Phys. Rev. C 60, 055803 (1999).

[18] L. Trache, A. Azhari, H.L. Clark, C.A. Gagliardi, Y.-W. Lui, A.M. Mukhamedzhanov, R.E. Tribble, and F. Carstoiu, Phys. Rev. C 58, 2715 (1998).

[19] P. Bém, V. Burjan, V. Kroha, J. Novák, S̆. Piskor̆, E. S̆imečková, J. Vincour, C.A. Gagliardi, A.M. Mukhamedzhanov, and R.E. Tribble, Phys. Rev. C 62, 024320 (2000).

[20] A.M. Mukhamedzhanov, R.P. Schmitt, R.E. Tribble, and A. Sattarov, Phys. Rev. C 52, 3483 (1995).

[21] C.R. Brune, W.H. Geist, R.W. Kavanagh, and K.D. Veal, Phys. Rev. Lett. 83, 4025 (1999).

[22] N. Imai et al., in Proceedings of the Conference of Nuclei in the Cosmos, Aarhus, Denmark, 2000, edited by J. ChristensenDalsgaard and K. Langanke (unpublished). 